\title{
A STUDY TO COMPARE THE CLINICAL FEATURES BETWEEN POST PULMONARY TUBERCULOSIS ASSOCIATED CHRONIC OBSTRUCTIVE PULMONARY DISEASE (COPD) WITH OTHER COPD PATIENTS
}

\author{
Saurabh Aggarwal11, Brijesh Kumar², Sanjay Kumar Verma³, Darshan Bajaj', Anand Kumar5, Surya Kant', Rajendra Prasad7 , \\ Ashok Kumar Singh ${ }^{8}$ \\ ${ }^{1}$ Assistant Professor, Department of Internal Medicine, GSVM Medical College, Kanpur, India. \\ ${ }_{2}^{2}$ Associate Professor, Department of Internal Medicine, GSVM Medical College, Kanpur, India. \\ ${ }_{3}^{3}$ Associate Professor, Department of TB \& Respiratory Diseases, Government Medical College, Kannauj, UP. \\ ${ }^{4}$ Associate Professor, Department of Pulmonary Medicine, King George's Medical University, Uttar Pradesh (Lucknow). \\ ${ }^{5}$ Associate Professor, Department of TB \& Respiratory Diseases, GSVM Medical College, Kanpur, India. \\ ${ }^{6}$ Professor and Head, Department of Pulmonary Medicine, King George's Medical University, Uttar Pradesh (Lucknow). \\ 7Professor and Head, Department of Pulmonary Medicine, Era's Lucknow Medical College, Lucknow, India. \\ ${ }^{8}$ Senior Consultant, Department of Critical Care Medicine and Respiratory Diseases, Regency Hospital Limited, Kanpur.
}

\section{ABSTRACT}

\section{BACKGROUND}

India is the highest TB burden country in the world and patients with treated TB may remain life-long sufferers of disabling sequelae. Many studies have shown that treated pulmonary tuberculosis can result in airflow obstruction. This study was designed to analyse the risk of Tuberculosis associated with Chronic obstructive pulmonary disease (COPD) among Chronic obstructive pulmonary disease patients.

\section{MATERIALS AND METHODS}

The patients of stable Chronic Obstructive Pulmonary Diseases will be picked up from outpatient and inpatient sections of the hospital at random. These COPD patients were divided into TB-associated COPD (only if their symptom of COPD started after the episode of pulmonary TB) and non-tubercular COPD group.

\section{RESULTS}

One hundred and thirty eight patients with COPD were enrolled during the study period. There were 107 males (77.5\%) and 31 (22.4\%) females. Of the total COPD patients, 58 (42.03\%) had past history of pulmonary tuberculosis treatment. TB associated COPD were younger in age as compared to non-TB COPD group. This study also revealed that clinical symptoms of COPD are similar in both groups, except high percentage of haemoptysis in TB associated COPD group. On comparison, FEV1/ FVC ratio in both groups were almost similar.

\section{CONCLUSION}

The present study highlighted that pulmonary tuberculosis is definitely associated with pulmonary damage and constitutes a significant proportion of COPD patients.

\section{KEYWORDS}

Pulmonary Tuberculosis, Airflow Obstruction, Chronic Obstructive Pulmonary Disease.

HOW TO CITE THIS ARTICLE: Aggarwal S, Kumar B, Verma SK, et al. A study to compare the clinical features between post pulmonary tuberculosis associated chronic obstructive pulmonary disease (COPD) with other COPD patients. J. Evolution Med. Dent. Sci. 2017;6(95):7015-7019, D0I: $10.14260 /$ jemds/2017/1522

\section{BACKGROUND}

Chronic obstructive pulmonary disease is a common, preventable and treatable disease that is characterised by persistent respiratory symptoms and airflow limitation that is due to airway and/ or alveolar abnormalities, usually caused by significant exposure to noxious particles or gases. ${ }^{1}$

Chronic obstructive pulmonary disease (COPD) is estimated to affect 65 million people worldwide and it is currently the third leading cause of death, accounting for approximately 3 million deaths annually.

'Financial or Other Competing Interest': None.

Submission 03-10-2017, Peer Review 06-12-2017,

Acceptance 12-12-2017, Published 23-12-2017.

Corresponding Author:

Dr. Brijesh Kumar,

Associate Professor,

Department of Internal Medicine,

GSVM Medical College, Kanpur, India.

E-mail: drbrijeshkumar74@gmail.com

DOI: $10.14260 /$ jemds $/ 2017 / 1522$

\section{(c) $(1)(5)$}

Ninety percent of these deaths are in low- and middleincome countries, where the prevalence of pulmonary tuberculosis (PTB) remains high. ${ }^{2-3}$

Cigarette smoking is the leading environmental risk factor for COPD and fewer than 50\% develop COPD during their lifetime. The other major risk factors are outdoor, occupational and indoor air pollution (resulting from the burning of wood and other biomass fuels). ${ }^{1}$

In the executive summary of the 2006 update of the Global Initiative for Chronic Obstructive Lung Disease (GOLD) guidelines, the role of tuberculosis in the development of chronic airways obstruction has been recognised. ${ }^{4}$ According to the GOLD Workshop summary, chronic bronchitis or bronchiolitis and emphysema can occur as complications of pulmonary tuberculosis. ${ }^{5}$

Treated post tubercular patients are left with permanent changes in lung anatomy and are at higher risk of pulmonary sequelae and premature mortality. ${ }^{6-9}$ These result in pulmonary sequelae that are characterised by bronchial and parenchymal structural changes including bronchovascular 
distortion, bronchiectasis, emphysematous changes and fibrotic bands. Moreover, these changes remain permanently in the lungs after a microbiological cure. ${ }^{10}$ The present study was done to know the prevalence of tuberculosis associated chronic obstructive pulmonary disease among total COPD patients and to compare the clinical features between post pulmonary tuberculosis associated COPD with other COPD patients.

\section{MATERIALS AND METHODS}

We conducted a cross-sectional study in the Department of Internal Medicine and Department of Tuberculosis and Respiratory Diseases, GSVM Medical College, Kanpur, Uttar Pradesh, from July 2016 to June 2017. The patients of stable Chronic Obstructive Pulmonary Diseases will be picked up from outpatient and inpatient sections of the hospital at random

\section{Inclusion Criteria}

COPD patients who are clinically stable will be included in the study.

\section{Exclusion Criteria}

1. Severe congestive heart failure.

2. Other respiratory diseases such as asthma, interstitial lung diseases, obstructive sleep apnoea, acute infections and severe hepatic failure and uncontrolled comorbidities such as malignancy.

3. Systemic Hypertension and Diabetes Mellitus.

\section{Design of the Study}

A detailed history and physical examination was carried out for every subject who entered in the study as per the predesigned proforma. Patients were examined clinically and radiologically with a view to establish diagnosis and condition made as per Global Initiative for Chronic Obstructive Lung Disease (GOLD) guidelines. They were subjected to Haemoglobin, Total Leucocyte Count (TLC), Differential Leucocyte Count (DLC), Fasting and Postprandial blood sugar, serum creatinine, SGPT, Serum bilirubin, serum protein, serum albumin and spirometry. Echocardiography, ECG and other investigations if required will also be done.

Body mass index (BMI) (kg/ m2) of each patient was calculated by measuring weight and height. Dyspnoea was assessed by using the modified British Medical Research Council (mMRC) dyspnoea scale.

Data was compiled using Microsoft Excel and analysed using SPSS Statistics version 20.0. Categorical variables were analysed using percentage and chi-square test. Two tailed ' $\mathrm{p}$ ' value less than 0.05 was considered significant. Quantitative variables were analysed using mean, standard deviation and student ' $\mathrm{t}$ ' test.

\section{Spirometry}

Spirometry measures were done in every patient who had symptoms and signs suggestive of COPD along with history of exposure to risk factors. The diagnosis of COPD was confirmed by spirometry and they were allocated into stages as per criteria adopted and standardised by GOLD 2016. Spirometry was performed using techniques that meet published standards. Both FEV 1 and FVC were the largest value obtained from any of the three technically satisfactory curves and FVC and FEV 1 values in these curves did not vary by more than $5 \%$ or $100 \mathrm{~mL}$, whichever was greater. Bronchodilator reversibility testing was also performed by inhaled salbutamol $200 \mu \mathrm{g}$ by metered dose inhaler via spacer, after withholding inhaled short-acting bronchodilator for 6 hrs., long acting $\beta 2$ agonists for $12 \mathrm{hrs}$. and sustained-release theophylline for $24 \mathrm{hrs}$. Lung functions were again measured 10 - 15 minutes after the inhaled bronchodilator.

In present study 138 patients who have exertional dyspnoea with or without cough, expectoration and/ or having past history of pulmonary tuberculosis with complete antituberculosis therapy were included. Patients were labelled as TB-associated COPD only if their symptom of COPD started after the episode of pulmonary TB. The prevalence of TBassociated COPD group was calculated, and its different parameters were compared with rest of COPD patients.

\section{RESULTS}

This study included 138 patients, 107 males and 31 females and their age ranging from 29 - 78 years with a mean age of 54.3 years. The mean ages among tuberculosis associated COPD and non-tuberculosis associated COPD were 46.8 and 59.8 years respectively. The mean body mass index among tuberculosis associated COPD and non-tuberculosis associated COPD were 17.74 and 19.25 respectively. Majority of the patients among TB associated COPD group had minimal chest $\mathrm{x}$-ray changes (more U/L involvements and less fibrotic changes). The percentage of smoking among TB-COPD group and non-TB-COPD group were $58.62 \%(34 / 58)$ and $76.25 \%$ (61/80) respectively. Dyspnoea were associated in all the patients among both groups. The FEV1/ FVC ratio among TBCOPD group and non-TB-COPD group were 53.71 and 53.14 respectively. The mean FEV1 (percentage predicted) among TB-COPD group and non-TB-COPD group were 46.03 and 47.15 respectively. The mean $\mathrm{FVC}$ (percentage predicted) among TB-COPD group and non-TB-COPD group were 58.20 and 58.57 respectively. The other features are summarised in table number 1 and 2 .

\begin{tabular}{|c|c|c|c|}
\hline Sl. No. & $\begin{array}{c}\text { Interval between Tt Completion and } \\
\text { Dyspnoea Starting (In Years) }\end{array}$ & $\begin{array}{c}\text { Total Number of Cases } \\
\text { (N= 58) }\end{array}$ & \% \\
\hline 1 & $<1$ & 3 & $5.17 \%$ \\
\hline 2 & $1-2$ & 6 & $10.4 \%$ \\
\hline 3 & $2-3$ & 3 & $5.17 \%$ \\
\hline 4 & $3-4$ & 6 & $10.4 \%$ \\
\hline 5 & $4-5$ & 15 & $25.86 \%$ \\
\hline 6 & $>5$ & 25 & $43.10 \%$ \\
\hline \multicolumn{2}{|c|}{ Table 1 } \\
\hline
\end{tabular}




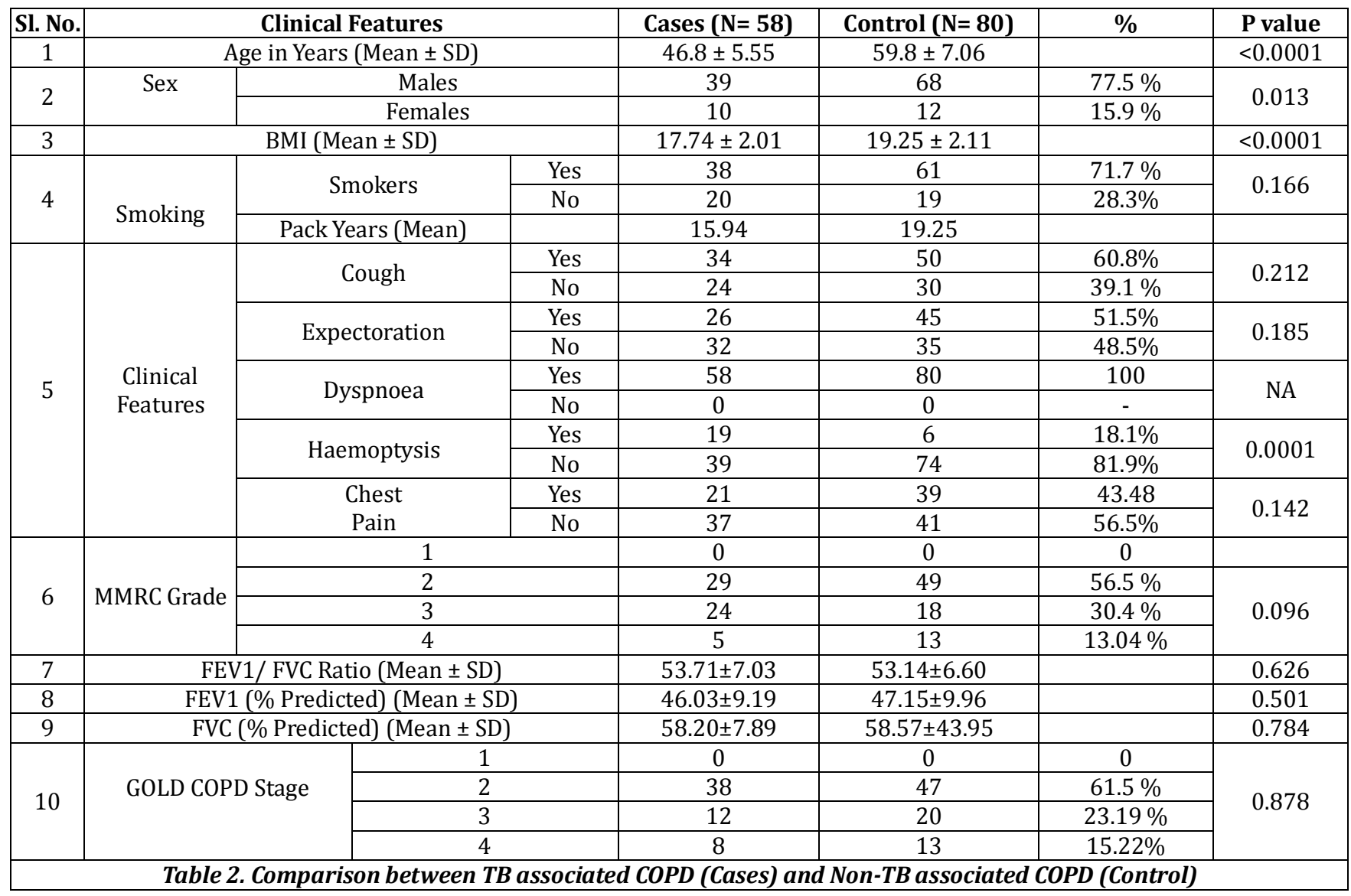

\section{DISCUSSION}

According to the global burden of disease projections, chronic obstructive pulmonary disease (COPD) and tuberculosis (TB) are among the 10 leading causes of death and disability for low- and middle-income countries. ${ }^{11}$

The result in present study showed that $42.03 \%$ cases of COPD had associated with tuberculosis in the past. A recent study from India was conducted to find out hospital-based prevalence of TB-associated COPD and their results showed that almost one-third of COPD patients (32.4\%) had associated TB in the past. 12 Another study from India which included 86 patients of COPD and showed that about $42 \%$ were associated with post pulmonary tuberculosis. ${ }^{13}$

A relationship between PTB and the development of COPD has been suggested in several reports. Willcox PA did a study to know the prevalence of chronic obstructive airways disease following treated pulmonary tuberculosis in seventy-one subjects who had previously been treated for tuberculosis. There was reported evidence of airways obstruction in 48 (68\%) patients. ${ }^{14}$

Plit ML et al did a study to know the influence of antimicrobial chemotherapy on spirometric parameters and pro-inflammatory indices in severe pulmonary tuberculosis. They recruited seventy-six patients into the study, of whom 74 completed the treatment programme. They found that improvement in lung function occurred in $54 \%$ of patients, but residual airflow limitation or a restrictive pattern was evident in $28 \%$ and $24 \%$ of patients, respectively. They also highlighted that antimicrobial chemotherapy may lead to improved lung function in patients with pulmonary tuberculosis, a large proportion of patients has residual impairment. ${ }^{15}$
Lee and Chang found that $\mathrm{CAO}$ is a common finding owing to TB-destroyed lung. ${ }^{16}$ Patricio Jiménez et al found that CAO is a common sequel with TB. 17 PLATINO study, a latest large population-based multicentre study, carried out in five Latin American countries ( $n=5571$ participants) included patients on the criteria of a past diagnosis of PTB by a physician and performed spirometry in the field. It included only those patients presenting to the hospital with dyspnoea. Along with the exclusion of other possible confounding factors, smokers and patients with age more than 65 years were also excluded. It was found that FEV1 is reduced compared with FVC in most cases. ${ }^{18}$ Our study also revealed similar results (FEV1 more reduced in comparison to FVC). However, another study had found that after 15 years follow-up of 40 patients, there was a higher yearly decline in FVC compared with FEV1.19

Lamprecht B analysed data from 14 countries from the International Burden of Obstructive Lung Disease (BOLD) study to describe characteristics of COPD in never smokers and to identify possible risk factors in this population. They reviewed a total of 10,000 subjects, who completed questionnaires and had acceptable post bronchodilator spirometry data and had information on smoking status. Of this group, 4,291 (42.9\%) were never the smokers. In their study, 5.3\% COPD patients had a history of the previous TB. ${ }^{20}$ While in present study, the numbers of TB associated COPD patients were on higher side. This is likely due to the fact that the study was done in never smokers, which excluded the causative effect of smoking in both COPD and TB. ${ }^{21-22}$ Moreover, it was a community-based study from low TB prevalence countries, which might have underscored the association between the two diseases. Ramakrishna $\mathrm{R}$ et al studied 100 cases of COPD diagnosed with FEV1/ FVC 0.7 or less presenting with symptoms of cough with expectoration 
and breathlessness and among them 57 (57\%) gave the history of tuberculosis. They also reported that age range of the patients was 41 to 81 . Average age of post tubercular airway disease was 58.2.23 While in present study, $42.03 \%$ had history of tuberculosis and mean age among TB associated COPD group was 46.8 years.

Different mechanisms have been proposed for the development of COPD in TB patients. It includes endobronchial involvement causing airway obstruction, bronchiolar narrowing and bronchiolitis obliterans resulting from peribronchial fibrosis and accelerated emphysematous changes caused by residual chronic or recurrent inflammation affecting lung compliance. ${ }^{24-25}$

The development and subsequent disease progression seen both in TB and COPD result in characteristic destructive parenchymal lung changes. A common link to the pathogenesis of both conditions may lie in the destruction of pulmonary Extracellular Matrix (ECM) comprising of collagen and elastin, which is the key to the structural integrity of the lung. ${ }^{26}$ The antigenic wall component of mycobacterium tuberculosis, lipoarabinomannan (LAM) stimulates the release of MMP-9 as well as up-regulating genetic expression of MMP-1 and MMP9. This not only results in the breakdown of collagen in the ECM, but also stimulates further lung damage by activation of other immune mediators such as Interleukin-8 and other cytokines. Hrabec et al demonstrated that MMP-9 levels were three times higher in the serum of tuberculosis patients compared with controls with increasingly elevated levels seen in those patients exhibiting advanced disease compared to limited disease. ${ }^{27}$ TNF alpha, which plays an important role in the formation of tuberculous granulomas has been demonstrated to stimulate MMP-9 secretion from granulomas cultured from patients with active tuberculosis. ${ }^{28}$

Risk of COPD has been found to increase with increase in radiological extent of $\mathrm{TB}$, increase in number of previous $\mathrm{TB}$ episodes as well as with delay in initiating anti-TB therapy. ${ }^{14,29-30}$ A study from India reported that around $90 \%$ of patients with TB-associated COPD had a history of a single episode of TB as well as had minimal CXR changes (unilateral involvement and apical linear fibrotic strands). ${ }^{12}$ Our study results are comparable to the above-mentioned study.

Lee SW did a study to know the Risk of Obstructive Lung Disease by Previous Pulmonary Tuberculosis in a Country with Intermediate Burden of Tuberculosis. Among 9,243 subjects, 3,687 enrolled for analysis and radiologists concluded that 294 $(8.0 \%)$ subjects were classified as having TB lesion on chest $\mathrm{x}-$ ray. All TB lesions were classified as inactive and there was no subject with lesion indicative of active TB on chest x-ray. Two hundred and ninety subjects had minimal lesions and four subjects had moderately or far-advanced lesions. This study also showed lower FEV1 in TB patients with minimal chest $\mathrm{x}$ ray changes. ${ }^{31}$ Our study results are comparable to the abovementioned study.

\section{CONCLUSION}

In the present study, we have evaluated the Tuberculosis associated COPD and compared with non-TB associated COPD group. To the best of our knowledge, such types of comparative studies have been published in lesser number. The present study highlighted that TB associated COPD were younger in age as compared to non-TB COPD group. This study also revealed that clinical symptoms of COPD are similar in both groups, except high percentage of haemoptysis in TB-COPD group. Our data also suggested that adequate durations of TB treatment might also help in reducing the prevalence of Tuberculosis associated COPD.

\section{REFERENCES}

[1] Global Initiative for Chronic Obstructive Lung Disease (GOLD): GOLD 2017 global strategy for the diagnosis, management, and prevention of chronic obstructive pulmonary disease, 2017 report. November 17, 2016. http://goldcopd.org/gold-2017-global-strategydiagnosis-management-preve.

[2] Lozano R, Naghavi M, Foreman $\mathrm{K}$, et al. Global and regional mortality from 235 causes of death for 20 age groups in 1990 and 2010: a systematic analysis for the global burden of disease study 2010. Lancet 2012;380(9859):2095-128.

[3] WHO: burden of chronic obstructive pulmonary disease.

http://www.who.int/respiratory/copd/burden/en/in dex.html (accessed August 29, 2011).

[4] Rabe KF, Hurd S, Anzueto A, et al. Global strategy for the diagnosis, management, and prevention of chronic obstructive pulmonary disease: GOLD executive summary. Am J Respir Crit Care Med 2007;176(6):53255.

[5] Pauwels RA, Buist AS, Carverley PM, et al. Global strategy diagnosis, management and prevention of chronic obstructive pulmonary disease. NHLBI/WHO global initiative for chronic obstructive lung disease (GOLD) workshop summary. Am J Respir Crit Care Med 2001;163(5):1256-76.

[6] Long R, Maycher B, Dhar A, et al. Pulmonary tuberculosis treated with directly observed therapy: serial changes in lung structure and function. Chest 1998;113(4):933-43.

[7] Rajasekaran S, Savithri S, Jeyaganesh D. Post tuberculosis bronchial asthma. Ind J Tub 2001;48:13942.

[8] De Valliere S, Barker RD. Residual lung damage after completion of treatment for multidrug-resistant tuberculosis. Int J Tuberc Lung Dis 2004;8(6):767-71.

[9] Yasuda J, Okada O, Kuriyama T, et al. Investigation of pulmonary hemodynamics and chest X-ray findings in patients with pulmonary tuberculosis sequelae and obstructive ventilator impairment. Kekkaku 1999;74:518.

[10] American Thoracic Society. Treatment of tuberculosis. MMWR Recomm Rep 2003;52(RR-11):1-77.

[11] Lopez AD, Mathers CD, Ezzati M, et al. Global and regional burden of disease and risk factors, 2001: systematic analysis of population health data. Lancet 2006;367(9524):1747-57.

[12] Aggarwal D, Gupta A, Janmeja AK, et al. Evaluation of tuberculosis-associated chronic obstructive pulmonary disease at a tertiary care hospital: a case-control study. Lung India 2017;34(5):415-9. 
[13] Ramavatar B, Saini AK, Kasana RK, et al. Assessment of airflow obstruction in post-tubercular COPD patients and non-tubercular COPD patients: a comperative study. Journal of Dental and Medical Sciences 2016;15(10):96-100.

[14] Willcox PA, Ferguson AD. Chronic obstructive airways disease following treated pulmonary tuberculosis. Respir Med 1989;83(3):195-8.

[15] Plit ML, Anderson R, Van Rensburg CE, et al. Influence of antimicrobial chemotherapy on spirometric parameters and pro-inflammatory indices in severe pulmonary tuberculosis. Eur Respir J 1998;12(2):3516.

[16] Lee JH, Chang JH. Lung functions in patients with chronic airflow obstruction due to tuberculous destroyed lung. Respir Med 2003;97(11):1237-42.

[17] Patricio Jiménez P, Vivianne Torres G, Paula Lehmann F, et al. Chronic airways obstruction in patients with tuberculosis sequel: a comparison with COPD. Rev Chil Enf Respir 2006;22:98-104.

[18] Menezes AM, Hallal PC, Perez-Padilla R, et al. Tuberculosis and airflow obstruction: evidence from the PLATINO study in Latin America. Eur Respir J 2007;30(6):1180-5.

[19] Vargha G. Fifteen-year follow-up of lung function in obstructive and non-obstructive pulmonary tuberculosis. Acta Med Hung 1983;40(4):271-6.

[20] Lamprecht B, McBurnie MA, Vollmer WM, et al. COPD in never smokers: results from the population-based burden of obstructive lung disease study. Chest 2011;139(4):752-63.

[21] Lin HH, Ezzati M, Murray M. Tobacco smoke, indoor air pollution and tuberculosis: a systematic review and meta-analysis. PLoS Med 2007;4(1):e20.

[22] Davies PD, Yew WW, Ganguly D, et al. Smoking and tuberculosis: the epidemiological association and immunopathogenesis. Trans R Soc Trop Med Hyg 2006;100(4):291-8.
[23] Ramakrishna R, Kumar PVK. Tuberculosis airway disease and bronchiectasis-a debilitating trio. J Evid Based Med Health 2016;3(20):818-22.

[24] Allwood BW, Myer L, Bateman ED. A systematic review of the association between pulmonary tuberculosis and the development of chronic airflow obstruction in adults. Respiration 2013;86(1):76-85.

[25] Chakrabarti B, Calverley PM, Davies PD. Tuberculosis and its incidence, special nature, and relationship with chronic obstructive pulmonary disease. Int J Chron Obstruct Pulmon Dis 2007;2(3):263-72.

[26] Elkington PT, Emerson JE, Lopez-Pascua LD, et al. Mycobacterium tuberculosis up-regulates matrix metalloproteinase-1 secretion from human airway epithelial cells via a p38 MAPK switch. J Immunol 2005;175(8):5333-40.

[27] Hrabec E, Strek M, Zieba M, et al. Circulation level of matrix metalloproteinase- 9 is correlated with disease severity in tuberculosis patients. Int J Tuberc Lung Dis 2002;6(8):713-9.

[28] Price NM, Gilman RH, Uddin J, et al. Unopposed matrix metalloproteinase- 9 expression in human tuberculous granuloma and the role of TNF-alpha-dependent monocyte networks. J Immunol 2003;171(10):557986.

[29] Hnizdo E, Singh T, Churchyard G. Chronic pulmonary function impairment caused by initial and recurrent pulmonary tuberculosis following treatment. Thorax 2000;55(1):32-8.

[30] Lee CH, Lee MC, Lin HH, et al. Pulmonary tuberculosis and delay in anti-tuberculous treatment are important risk factors for chronic obstructive pulmonary disease. PLoS One 2012;7(5):e37978.

[31] Lee SW, Kim YS, Kim DS, et al. The risk of obstructive lung disease by previous pulmonary tuberculosis in a country with intermediate burden of tuberculosis. J Korean Med Sci 2011;26(2):268-73. 